\title{
Multiple $\zeta$-motives and moduli spaces $\overline{\mathcal{M}}_{0, n}$
}

\author{
A. B. Goncharov and Yu. I. Manin
}

\begin{abstract}
We give a natural construction of framed mixed Tate motives unramified over $\mathbb{Z}$ whose periods are the multiple $\zeta$-values. Namely, for each convergent multiple $\zeta$-value we define two boundary divisors $A$ and $B$ in the moduli space $\overline{\mathcal{M}}_{0, n+3}$ of stable curves of genus zero. The corresponding multiple zeta-motive is the $n$th cohomology of the pair $\left(\overline{\mathcal{M}}_{0, n+3}-A, B\right)$.
\end{abstract}

\section{Introduction and summary}

\section{$1.1 \zeta$-values and moduli spaces}

i) The multiple $\zeta$-values were defined by Euler [Eul17] as the series

$$
\zeta\left(n_{1}, \ldots, n_{m}\right)=\sum_{0<k_{1}<\cdots<k_{m}} \frac{1}{k_{1}^{n_{1}} \cdots k_{m}^{n_{m}}}, \quad n_{m}>1 .
$$

ii) Recall that $\overline{\mathcal{M}}_{0, n+3}$ parametrizes stable curves of genus zero with $n+3$ labeled points. It is stratified according to the type of degeneration. The open stratum $\mathcal{M}_{0, n+3}$ can be identified with $\left(\mathbf{P}^{1}\right)^{n+3}$ minus diagonals modulo Aut $\mathbf{P}^{1}$. Then

$$
\partial \overline{\mathcal{M}}_{0, n+3}:=\overline{\mathcal{M}}_{0, n+3}-\mathcal{M}_{0, n+3}
$$

is a normal crossing divisor, and the pair $\left(\overline{\mathcal{M}}_{0, n+3}, \partial \overline{\mathcal{M}}_{0, n+3}\right)$ is defined over $\mathbb{Z}$. We use it to construct a framed mixed Tate motive unramified over $\mathbb{Z}$ whose period is given by the multiple $\zeta$-value (1).

Generally, let $A$ and $B$ be unions of some irreducible components of $\partial \overline{\mathcal{M}}_{0, n+3}$. Suppose that no irreducible component is shared by $A$ and $B$. Then we show that

$$
H^{n}\left(\overline{\mathcal{M}}_{0, n+3}-A, B-A \cap B\right)
$$

is a mixed Tate motive unramified over $\mathbb{Z}$. A choice of two non-zero elements

$$
\left[\Omega_{A}\right] \in \operatorname{Gr}_{2 n}^{W} H^{n}\left(\overline{\mathcal{M}}_{0, n+3}-A\right), \quad\left[\Delta_{B}\right] \in\left(\operatorname{Gr}_{0}^{W} H^{n}\left(\overline{\mathcal{M}}_{0, n+3}, B\right)\right)^{\vee}
$$

defines a framed mixed Tate motive unramified over $\mathbb{Z}$ given by the triple

$$
\left(H^{n}\left(\overline{\mathcal{M}}_{0, n+3}-A, B_{A}\right) ;\left[\Omega_{A}\right],\left[\Delta_{B}\right]\right) .
$$

For every multiple $\zeta$-value (1) we construct two divisors on $\overline{\mathcal{M}}_{0, n+3}$ as above such that the period of the corresponding motive is this value.

Another construction of the multiple polylogarithm motives has been given in [Gon02, ch. 3]. The construction given here differs from the earlier one for multiple $\zeta$-values.

Received 16 April 2002, accepted in final form 24 October 2002.

2000 Mathematics Subject Classification 11G55, 11F67, 11R32, $20 \mathrm{~F} 34$.

Keywords: multiple $\zeta$-values, moduli spaces, mixed Tate motives.

The first author was supported by the NSF grant DMS-0099390.

This journal is (c) Foundation Compositio Mathematica 2004. 


\section{A. B. Goncharov and Yu. I. Manin}

\subsection{A sketch of the construction of multiple $\zeta$-motives}

First, let us recall how to represent multiple $\zeta$-values by iterated integrals. Starting with positive integers $n_{1}, \ldots, n_{m}$ as in (1), we put $n:=n_{1}+\cdots+n_{m}$, and $\underline{\varepsilon}:=\left(\varepsilon_{1}, \ldots, \varepsilon_{n}\right)$ where $\varepsilon_{i}=0$ or 1 , and $\varepsilon_{i}=1$ precisely when $i \in\left\{1, n_{1}+1, n_{1}+n_{2}+1, \ldots, n_{1}+\cdots+n_{m-1}+1\right\}$. Furthermore, put

$$
\omega(\underline{\varepsilon}):=\frac{d t_{1}}{t_{1}-\varepsilon_{1}} \wedge \cdots \wedge \frac{d t_{n}}{t_{n}-\varepsilon_{n}}
$$

and

$$
\Delta_{n}^{0}:=\left\{\left(t_{1}^{0}, \ldots, t_{n}^{0}\right) \in \mathbb{R}^{n} \mid 0<t_{1}^{0}<\cdots<t_{n}^{0}<1\right\} .
$$

By the Leibniz-Kontsevich formula (cf. [Gon01b, Theorem 2.2])

$$
\zeta\left(n_{1}, \ldots, n_{m}\right)=\zeta(\underline{\varepsilon})=(-1)^{m} \int_{\Delta_{n}^{0}} \omega(\underline{\varepsilon}) .
$$

The integral converges because $\varepsilon_{1} \neq 0, \varepsilon_{n} \neq 1$.

Let us identify points of $\mathcal{M}_{0, n+3}(\mathbb{C})$ with sequences $\left(t_{1}^{0}, \ldots, t_{n}^{0}\right) \in \mathbb{C}^{n}$ such that $t_{i}^{0} \neq 0,1, t_{i}^{0} \neq t_{j}^{0}$ for $i \neq j$, using the rule

$$
\left\{0, t_{1}^{0}, \ldots, t_{n}^{0}, 1, \infty\right\} \bmod \text { Aut } \mathbf{P}^{1} \Leftrightarrow\left(t_{1}^{0}, \ldots, t_{n}^{0}\right) .
$$

Moreover, consider the form $\omega(\underline{\varepsilon})$ as a holomorphic form on $\mathcal{M}_{0, n+3}$. Meromorphically extending it to $\overline{\mathcal{M}}_{0, n+3}$ we get a form $\Omega(\underline{\varepsilon})$ with logarithmic singularities at the boundary $\partial \overline{\mathcal{M}}_{0, n+3}$. Finally, we identify the open simplex $\Delta_{n}^{0}$ with its image $\Phi\left(\Delta_{n}^{0}\right)$ in $\mathcal{M}_{0, n+3}$. Then we obviously have

$$
\zeta\left(n_{1}, \ldots, n_{m}\right)=(-1)^{m} \int_{\Phi\left(\Delta_{n}^{0}\right)} \Omega(\underline{\varepsilon}) .
$$

Now let us introduce divisors $A=A(\underline{\varepsilon})$ and $B$ and interpret this integral as a period of a framed mixed Hodge structure (cf. [Gon99, § 4] and $\S 4$ ).

The divisor $A(\underline{\varepsilon})$. It is the divisor of singularities of the form $\Omega(\underline{\varepsilon})$.

The divisor $B$. Let $\bar{\Phi}_{n}$ be the closure of the open simplex $\Phi\left(\Delta_{n}^{0}\right)$. We define $B$ to be the Zariski closure of the boundary of $\bar{\Phi}_{n}$. Thus there is a relative homology class

$$
\left[\bar{\Phi}_{n}\right] \in H_{n}\left(\overline{\mathcal{M}}_{0, n+3}(\mathbb{C}), B(\mathbb{C}) ; \mathbb{Z}\right) .
$$

In $\S \S 2$ and 3 we will clarify the structure of arbitrary choices involved in these constructions. Moreover, we will explicitly compute $B$ and $A(\underline{\varepsilon})$, in terms of the standard combinatorial description of boundary components of $\partial \overline{\mathcal{M}}_{0, n+3}$.

The closed cell $\bar{\Phi}_{n}$ is naturally identified with a Stasheff polytope. The divisor $B$ is an algebraic counterpart of the Stasheff polytope. Specifically, we show that, intersecting a $k$-dimensional stratum of the divisor $B$ with the cell $\bar{\Phi}_{n}$, we get a real $k$-dimensional face of this cell. This face is naturally identified with the corresponding face of the Stasheff polytope. This fact together with convergence of the integral (5) immediately imply the following key lemma.

Lemma 1.1. The divisors $A(\underline{\varepsilon})$ and $B$ share no common irreducible components.

In $\S 3$ we will directly check this lemma using our combinatorial calculations.

Lemma 1.1 guarantees that we can set

$$
\zeta^{\mathcal{M}}\left(n_{1}, \ldots, n_{m}\right):=H^{n}\left(\overline{\mathcal{M}}_{0, n+3}-A(\underline{\varepsilon}), B-B \cap A(\underline{\varepsilon})\right) .
$$

We show in $\S 4$ that the form $\Omega(\underline{\varepsilon})$ and the cycle $\bar{\Phi}_{n}$ provide a framing, and the period of the corresponding mixed Hodge structure is given by (1). The fact that this period is a well-defined number reflects the following strengthening of Lemma 1.1: the boundary of $\Phi_{n}$ does not intersect $A(\underline{\varepsilon})$ (see Corollary 3.2). 


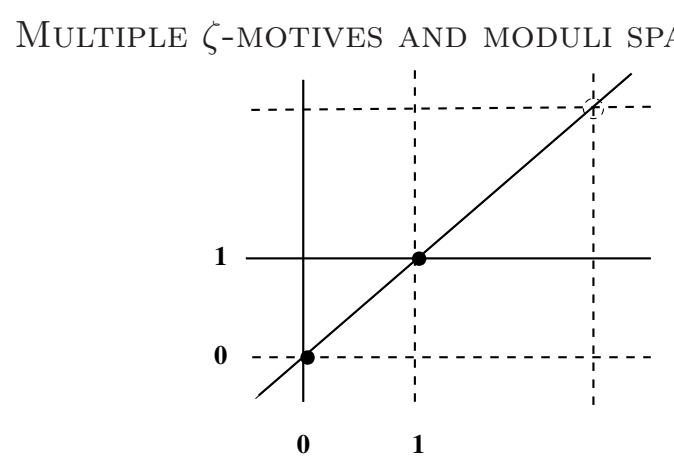

FiguRE 1.

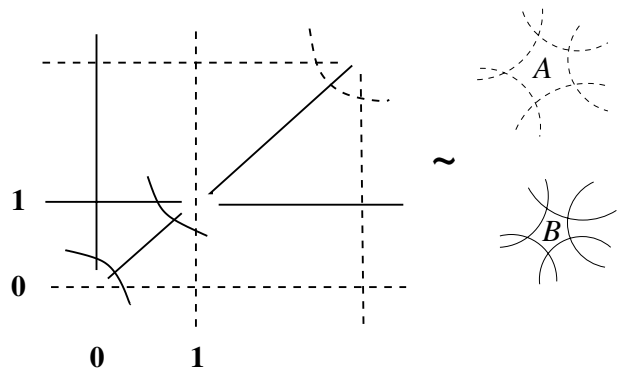

FigURE 2.

\subsection{The simplest example: $\zeta^{\mathcal{M}}(2)$}

The integral representation for $\zeta(2)$ is given by the following formula discovered by Leibniz:

$$
\zeta(2)=\iint_{0<t_{1}<t_{2}<1} \frac{d t_{1}}{1-t_{1}} \wedge \frac{d t_{2}}{t_{2}} .
$$

Let us consider first $\left(t_{1}, t_{2}\right)$ as the affine coordinates on $\mathbf{P}^{1} \times \mathbf{P}^{1}$. Then the singularities of the integrand provide a rectangle $\mathcal{A}$ given by the punctured lines in Figure 1, whereas the Zariski closure of the boundary of the integration cycle is the bold triangle $\mathcal{B}$ in the figure.

The moduli space $\overline{\mathcal{M}}_{0,5}$ can be obtained by blowing up the three special points $(0,0),(1,1)$, $(\infty, \infty)$ on the diagonal - see the proof of Proposition 3.1. They are the points where the divisor $\mathcal{A} \cup \mathcal{B}$ (the union of the seven lines in Figure 1) fails to be a normal crossing divisor.

Thus we can identify $\overline{\mathcal{M}}_{0,5}$ with a del Pezzo surface of degree five. The boundary $\partial \overline{\mathcal{M}}_{0,5}$ is given by the ten exceptional curves on it. They are the strict preimages of the seven lines on $\mathbf{P}^{1} \times \mathbf{P}^{1}$ plus the three curves blown down by the projection onto $\mathbf{P}^{1} \times \mathbf{P}^{1}$. The divisors $A=A(1,0)$ and $B$ are given by two complementary algebraic pentagons (see Figure 2). The union of these two pentagons is the whole boundary $\overline{\mathcal{M}}_{0,5}$. Such a decomposition of the boundary into a union of two pentagons is not unique, but the automorphism group $S_{5}$ acts transitively on the set of all decompositions.

We define the $\zeta^{\mathcal{M}}(2)$-motive as $H^{2}\left(\overline{\mathcal{M}}_{0,5}-A, B-B \cap A\right)$. The cell $\bar{\Phi}_{2}$ is a pentagon. It provides a class $\left[\bar{\Phi}_{2}\right]$ in the Betti realization of $H_{2}\left(\overline{\mathcal{M}}_{0,5}-A, B-B \cap A\right)$. The form $\Omega(1,0)$ gives a class in the De Rham realization of $\zeta^{\mathcal{M}}(2)$, and the number $\zeta(2)$ equals the pairing $\left\langle\Omega(1,0),\left[\bar{\Phi}_{2}\right]\right\rangle$.

Remark. It might be tempting to consider the simpler motive $H^{2}\left(\mathbf{P}^{1} \times \mathbf{P}^{1}-\mathcal{A}, \mathcal{B}-\mathcal{B} \cap \mathcal{A}\right)$. However, $\zeta(2)$ is not its period. Indeed, removing $\mathcal{A}$ we delete a vertex $(0,0)$ of the closed triangle $\Delta_{2}$. Thus $\Delta_{2}$ does not provide an element in the Betti realization of $H_{2}\left(\mathbf{P}^{1} \times \mathbf{P}^{1}-\mathcal{A}, \mathcal{B}-\mathcal{B} \cap \mathcal{A}\right)$. 


\section{A. B. Goncharov and Yu. I. Manin}

\section{Geometry of $\overline{\mathcal{M}}_{0, n+3}$ and divisors $B$}

In this section we give a summary of the properties of $\overline{\mathcal{M}}_{0, n+3}$ which will be used in the next section and calculate the divisor $B$.

\subsection{Reminder on moduli spaces}

Let $S$ be any finite set, $|S| \geqslant 3$. We recall that $\overline{\mathcal{M}}_{0, S}$ carries a universal family of stable curves of genus zero, $\pi_{S}: \overline{\mathcal{C}}_{0, S} \rightarrow \overline{\mathcal{M}}_{0, S}$ endowed with structure sections labeled by $S, x_{s}: \overline{\mathcal{M}}_{0, S} \rightarrow \overline{\mathcal{C}}_{0, S}$, $s \in S$. Hence geometric points of $\overline{\mathcal{M}}_{0, S}$ can be identified with stable $S$-labeled pointed curves.

For any $S^{\prime} \subset S,\left|S^{\prime}\right| \geqslant 3$, there is a canonical forgetful morphism $\phi_{T}: \overline{\mathcal{M}}_{0, S} \rightarrow \overline{\mathcal{M}}_{0, S^{\prime}}, T:=S-S^{\prime}$. In terms of geometric points, $\phi_{T}$ forgets all sections marked by $s \in T$ and then contracts the unstable components.

Morphisms forgetting disjoint subsets of points commute in an obvious sense, i.e. functorial on inclusions $S^{\prime} \subset S$, and their product is a forgetful morphism forgetting the union of these subsets.

In particular, if $T=\{t\}$, we get two morphisms with common base: $\phi_{\{t\}}$ and $\pi_{S^{\prime}}: \overline{\mathcal{C}}_{0, S^{\prime}} \rightarrow \overline{\mathcal{M}}_{0, S^{\prime}}$. A basic fact in the theory of moduli spaces is the existence of the canonical isomorphism

$$
\mu_{S}: \overline{\mathcal{C}}_{0, S^{\prime}} \cong \overline{\mathcal{M}}_{0, S}
$$

transforming $\phi_{\{t\}}$ to $\pi_{S^{\prime}}$.

\subsection{Combinatorics of boundary strata}

The space $\overline{\mathcal{M}}_{0, S}$ is stratified by locally closed subschemes $\mathcal{M}_{\tau}$ indexed by the (isomorphism classes of the) stable trees with tails labeled by $S$, which are the dual graphs of the respective stable curves. Closures of all strata are smooth. The open stratum $\mathcal{M}_{0, S}$ parametrizes irreducible curves with inclusions $S \rightarrow \mathbf{P}^{1}$. Codimension one strata parametrize two-component curves and can be indexed by unordered 2-partitions $S=S^{\prime} \cup S^{\prime \prime}$ with $\left|S^{(i)}\right| \geqslant 2$ (stability), describing how the labeled points are distributed between the two components.

In what follows, we will often denote such a partition as $S^{\prime} \mid S^{\prime \prime}$ or similarly. For example, if $S=\{0,1, \infty, s\}$, then $0 \infty \mid 1 s$ means that $S^{\prime}=\{0, \infty\}, S^{\prime \prime}=\{1, s\}$.

The total boundary is a normal crossing divisor. The closed strata of the boundary are strata of this divisor: each codimension- $d$ stratum is an intersection of exactly $d$ boundary divisors, and their set is unique. A combinatorial description of this picture, which we will need in the following section, is as follows.

Consider two stable 2-partitions of $S: \sigma:=\sigma_{1} \mid \sigma_{2}$ and $\tau:=\tau_{1} \mid \tau_{2}$. Put

$$
\delta(\sigma, \tau):=\left\{\text { the number of non-empty intersections } \sigma_{i} \cap \tau_{j} \text { minus } 2\right\} .
$$

This is a kind of distance: $\delta(\sigma, \tau)=2$ if and only if the respective divisors do not intersect; $\delta(\sigma, \tau)=1$, if and only if their intersection is of codimension two; $\delta(\sigma, \tau)=0$ if and only if they coincide.

A subset $\mathcal{D}$ of boundary divisors determines a non-empty stratum exactly when all pairwise distances between the respective partitions equal one. Knowing this subset, we can also reconstruct the tree $\tau$ describing the respective degeneration type: this is the maximal tree with tails labeled by $S$ such that if one cuts it at the middle of any internal edge, the resulting 2-partition of $S$ will belong to $\mathcal{D}$.

Moreover, knowing the tree $\tau$ we can determine the structure of the stratum $\mathcal{M}_{\tau}$ itself: it is canonically isomorphic to the product $\prod_{v \in V_{\tau}} \mathcal{M}_{0, F_{\tau}(v)}$ where $V_{\tau}$ is the set of vertices, and $F_{\tau}(v)$ is the set of flags incident to $v$. 


\section{Multiple $\zeta$-Motives And moduli spaces $\overline{\mathcal{M}}_{0, n}$}

To determine the image of the boundary divisor defined by a partition of $S$ under the forgetful morphism $\phi_{T}: \overline{\mathcal{M}}_{0, S} \rightarrow \overline{\mathcal{M}}_{0, S^{\prime}}$, one should consider the induced partition of $S^{\prime}$. If it is stable, the respective divisor is the image. If it is unstable, the image is the total space $\overline{\mathcal{M}}_{0, S^{\prime}}$. For example, under the isomorphism (6), the $S^{\prime}$-labeled structure sections of $\overline{\mathcal{C}}_{0, S^{\prime}}$ correspond exactly to those boundary divisors $t s \mid S^{\prime}-\{s\}\left(s \in S^{\prime}\right)$ of $\overline{\mathcal{M}}_{0, S}$ which become unstable after forgetting $t$.

For proofs and further details, see [Knu83] and [Man99, ch. 3, § 3].

\subsection{Real points of $\overline{\mathcal{M}}_{0, S}$}

Consider a stable $S$-labeled curve over $\mathbb{C}$. Endowing it with the complex conjugate structure we will produce another similar curve. Thus we have a conjugation involution acting upon $\overline{\mathcal{M}}_{0, S}(\mathbb{C})$. Fixed points of this involution form the space of real points $\overline{\mathcal{M}}_{0, S}(\mathbb{R})$. A stable curve living over a real point is itself real in the following sense: it is endowed with a conjugation involution fixing all labeled points and all singular points. Thus, every irreducible component of a real stable labeled curve is a sphere $\mathbf{P}^{1}(\mathbb{C})$ endowed with a real equator $\mathbf{P}^{1}(\mathbb{R})$ carrying all labeled points and eventually all intersection points with other components.

Taken all together, such points are called special points. In the dual graph $\tau$, an irreducible component becomes a vertex, and special points become flags at this vertex. Thus, a real structure of the respective curve determines an additional structure on $\tau$ : an unoriented cyclic order on the set of flags at each vertex. To define it, just look at how the special points are distributed along the real equator of the respective component.

We will call a choice of such orders a locally planar structure on $\tau$. Varying the curve along one connected component of the intersection of $\overline{\mathcal{M}}_{0, S}(\mathbb{R})$ with the $\tau$-stratum does not change the local planar structure.

The following proposition furnishes additional details of this picture.

\section{Proposition 2.1.}

i) The set $\overline{\mathcal{M}}_{0, S}(\mathbb{R})$ is a connected closed real manifold. Connected components of intersections of $\overline{\mathcal{M}}_{0, S}(\mathbb{R})$ with complex boundary strata form a cell decomposition. Cells of it are in one-to-one correspondence with stable locally planar $S$-labeled trees. The relation 'a cell is a codimension one component of the boundary of another cell' corresponds to the relation 'a locally planar tree produces another locally planar tree by contracting an internal edge'.

ii) Fix an unoriented cyclic order on $S$ and consider the respective open cell. Any choice of three consecutive labels with respect to this order allows one to introduce real coordinates which identify the open cell with the simplex $\Delta_{n}^{0}$, where $|S|=n+3$. This identification was denoted $\Phi$ in (5).

iii) The closure of each open cell has the structure of a Stasheff polytope. In particular, its boundary strata of codimension one are indexed by those stable 2-partitions of $S$ which are compatible with the respective cyclic order: they correspond to breaking the real equator into two connected arcs.

iv) The Zariski closure of the boundary of any open cell is a union of boundary divisors having the same combinatorial shape ('an algebraic Stasheff polytope').

Sketch of a proof. One can extract a proof of this proposition from [Kap93] (cf. also [Dev99]). We will give here a modified version, including a differential geometric picture of the degeneration of stable curves which in particular makes it clear why Stasheff polytopes appear at all. Assume that $|S| \geqslant 4$.

Let us look first at the open cells. A contemplation will convince the reader that they are labeled by the cyclic orders $\rho$ of $S$ and that an arbitrary order can arise. To identify each cell with a simplex, we choose three consecutive labels with respect to $\rho$ and denote them $1, \infty$, and 0 . 


\section{A. B. Goncharov and Yu. I. Manin}

For any $s \in S-\{1, \infty, 0\}$, consider the forgetful morphism $\overline{\mathcal{M}}_{0, S} \rightarrow \overline{\mathcal{M}}_{\{0,1, \infty, s\}}$. The target space is a projective line with three special points: boundary divisors corresponding to the partitions $s 0 \mid 1 \infty$, $s 1 \mid 0 \infty$, and $s \infty \mid 01$.

We define $t_{s}$ to be the lift from $\overline{\mathcal{M}}_{0,\{0,1, \infty, s\}}$ of the affine coordinate on this projective line taking, respectively, the values 0,1 , and $\infty$ at these three boundary divisors.

Put $S-\{1, \infty, 0\}=\left\{s_{1}, \ldots, s_{n}\right\}$ where the labels are cyclically ordered so that $0<s_{1}<\cdots<$ $s_{n}<1<\infty<0$. From our definitions it follows that, with coordinates $t_{s_{i}}$, the interior part of the respective real component can be identified with the simplex $\Delta_{n}^{0}$ as in $\S 1$.

Now let us turn to the boundary of the closure of this simplex in $\overline{\mathcal{M}}_{0, S}(\mathbb{C})$. As we have already said, a point $z \in \mathcal{M}_{0, S}(\mathbb{C})$ corresponds to the Riemannian sphere $\mathbf{P}^{1}(\mathbb{C})$ with marked points labeled by $S$. If $z$ is real, this sphere has a distinguished equator $\mathbf{P}^{1}(\mathbb{R})$ carrying all marked points $x_{s}(z)$.

Endow $\mathbf{P}^{1}(\mathbb{C})-\left\{x_{s}(z) \mid s \in S\right\}$ with a hyperbolic metric of constant curvature -1 . The marked points will go to infinity as the ends of infinitely thinning tubes, and the whole picture will resemble a chestnut.

When $z$ tends to a point of a boundary divisorial stratum, the respective variable surface carries a closed geodesic whose length tends to zero, a representative of the vanishing 1-homology class. The body of the chestnut near the limit becomes divided into two new bodies, connected by a thin tube whose length tends to infinity as well. Some of the labeled points will belong to one half, the remainder will belong to the other half. This unordered 2-partition determines the relevant boundary component of the moduli space.

The key fact is this: if $z$ tends to the boundary in this way along a real path in $\mathcal{M}_{0, S}(\mathbb{C})$, then the vanishing geodesic intersects the real equator exactly at two points. When the vanishing cycle shrinks, the equator itself degenerates into a union of two real equators of the components of the limit curve. Hence the respective 2-partition of $S$ can be obtained by breaking the equator into two arcs (say, by drawing a chord).

From [Kap93] it follows that all such stable 2-partitions compatible with the cyclic order arise in this way. Geometrically, such a partition arises in the limit when all points of one part of the partition try to merge.

Strata of the real boundary of larger codimension (faces) can be obtained by iterating this description, i.e. applying it in turn to the components of a degenerate curve. In this way, these strata become indexed by 'cactiform' trees, whose 'edges' are circles carrying marked points. As in the complex case, we took the dual graphs and replaced these graphs by actual trees: vertices replace circles; halves of edges replace marked points; edges replace contact points of pairs of circles.

Equivalently, put the points labeled by $S$ on a circle in a plane compatibly with the chosen order. Strata of codimension $d$ of the closure of the respective component are in a natural bijection with the isotopy classes of stable trees with $d$ (internal) edges situated inside this circle and having the labeled points as ends of tails. A stratum of codimension $d+1$ belongs to the closure of the stratum of codimension $d$ if one can obtain the smaller tree from the larger one by collapsing one of its edges. The resulting polytope is called the Stasheff polytope $K_{n}$ (if $|S|=n+3$ ). Its vertices correspond to the trivalent trees.

Notice that in Proposition 2.1 we were speaking about locally planar trees whereas the picture above refers to a global planar embedding. The point is that any given cell belongs to the boundaries of many maximal cells. They are classified precisely by those planar embeddings which are compatible with a given locally planar structure.

Any face of a Stasheff polytope is a product of Stasheff polytopes (some of them may be points).

The Zariski closure $B$ of the boundary of any open cell is a union of boundary divisors having the same combinatorial shape as a Stasheff polytope $K$ in the following sense: there is a bijection 
Multiple $\zeta$-Motives And moduli spaces $\overline{\mathcal{M}}_{0, n}$
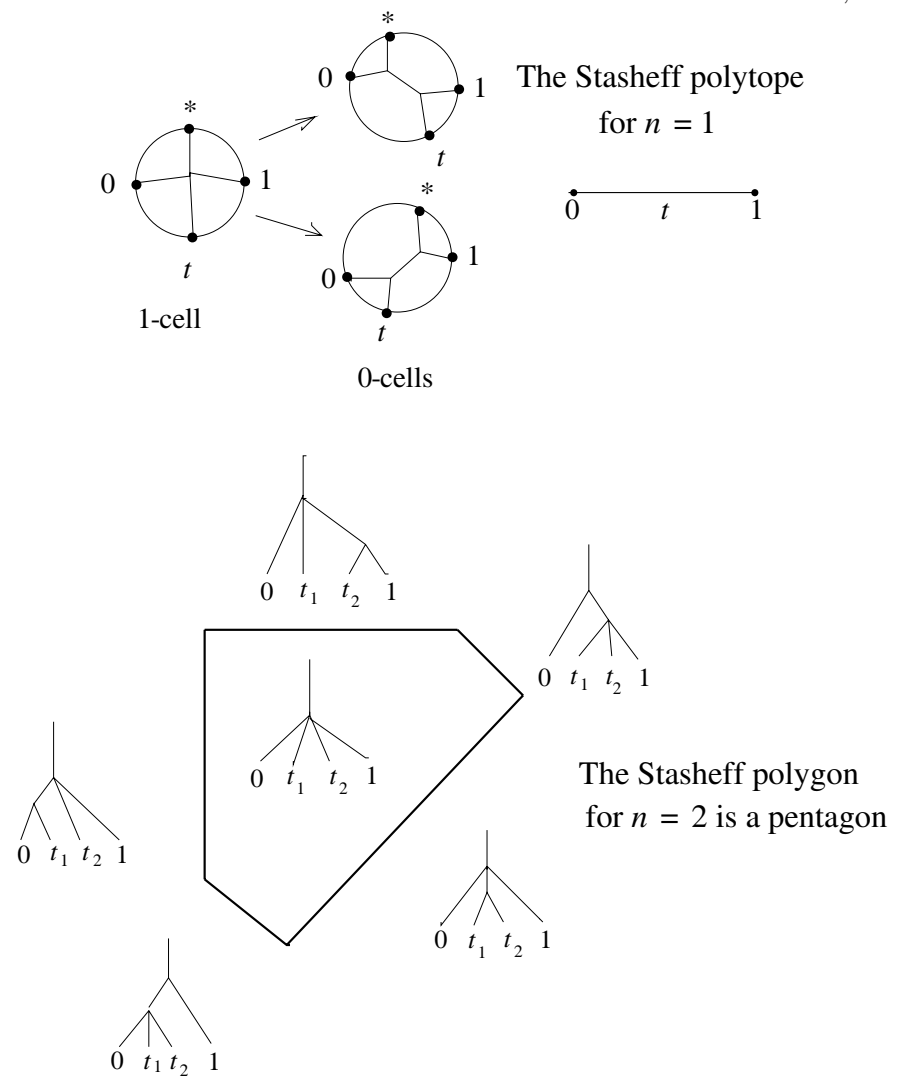

FIGURE 3.

between the irreducible components $B_{i}$ of $B$ and the codimension-one faces $F_{i}$ of $K$ such that a subset of components $B_{i}$ has a non-empty intersection of expected codimension if and only if the respective subset of faces $F_{i}$ has this property.

A more elementary example is that $n+1$ generic hyperplanes in $\mathbf{P}^{n}$ have the shape of a simplex. However, four lines in $\mathbf{P}^{2}$ do not have the shape of a convex quadrangle.

\subsection{Divisors $B(\rho)$}

It is convenient to repeat the formal definition of $B(\rho)$ : if $\rho$ is any cyclic order on $S, B(\rho)$ is the sum of boundary divisors corresponding to those 2-partitions that are provided by breaking the circle into two arcs. This divisor carries the total boundary of the respective open cell $\mathcal{M}_{0, S}^{\rho}(\mathbb{R})$.

\section{Forms $\Omega(\underline{\varepsilon})$ and divisors $A(\underline{\varepsilon})$}

\subsection{Construction of $\Omega(\underline{\varepsilon})$}

As above, choose and fix three labels in $S$ which we will denote by 0,1 , and $\infty$. We have shown that this choice allows us to define a meromorphic function $t_{s}$ on $\overline{\mathcal{M}}_{0, S}$ for each $s \in S-\{0,1, \infty\}$.

The additional structure is an arbitrary function $\underline{\varepsilon}: S-\{0,1, \infty\} \rightarrow\{0,1\}, s \mapsto \varepsilon_{s}$. Choosing an order of $S-\{0,1, \infty\}$, we can define a meromorphic form on $\overline{\mathcal{M}}_{0, S}$ :

$$
\Omega(\underline{\varepsilon}):=\bigwedge_{s \in S-\{0,1, \infty\}} \frac{d t_{s}}{t_{s}-\varepsilon_{s}} .
$$

Changing the order influences only the sign of the form. 


\section{A. B. Goncharov and Yu. I. Manin}

\subsection{Divisor $A(\underline{\varepsilon})$}

We continue using the notation as in (7).

For $\alpha \in\{0,1, \infty\}$, we define $S(\alpha)=S(\alpha, \underline{\varepsilon})$ by

$$
S(0):=\left\{s \mid \varepsilon_{s}=0\right\}, \quad S(1):=\left\{s \mid \varepsilon_{s}=1\right\}, \quad S(\infty):=S(0) \cup S(1) .
$$

We will say that a 2-partition of $S$ has type $\alpha$ with respect to $\underline{\varepsilon}$ if one part of it is of the form $\{\alpha\} \cup T$ where $T$ is a non-empty subset of $S(\alpha)$.

Proposition 3.1. The divisor of singularities of $\Omega(\underline{\varepsilon})$ on $\overline{\mathcal{M}}_{0, S}$ is the sum $A(\underline{\varepsilon})$ of boundary divisors corresponding to those stable 2-partitions of $S$ which have some type with respect to $\underline{\varepsilon}$.

The following result is stronger than Lemma 1.1. Denote by $\rho$ the cyclic order $0<s_{1}<\cdots<$ $s_{n}<1<\infty<0$.

Corollary 3.2. Assume that $n \geqslant 2$, and $\underline{\varepsilon}$ and $\rho$ satisfy $\varepsilon_{s_{1}}=1, \varepsilon_{s_{n}}=0$. Then $A(\underline{\varepsilon})$ does not intersect the boundary of $\mathcal{M}_{0, S}^{\rho}(\mathbb{R})$.

In particular, $\Omega(\underline{\varepsilon})$ is holomorphic on $\overline{\mathcal{M}}_{0, S}-A(\underline{\varepsilon})$, the closure of $\Phi\left(\Delta_{n}\right)$ determines a relative cycle on $\overline{\mathcal{M}}_{0, S}-A(\underline{\varepsilon})$ modulo $B(\rho) \cap A(\underline{\varepsilon})$, and formula (5) can be written as the integral over this relative cycle.

Deduction of the Corollary. Assume that the intersection in question is not empty. Since it is closed, it has to contain a zero-dimensional face of (the closure of) $\mathcal{M}_{0, S}^{\rho}(\mathbb{R})$. As was explained above, this face must correspond to a planar tree $\tau$ having all vertices of multiplicity three, and drawn inside the circle on which the labels $S$ are put in the order $\rho$ and mark the tails of $\tau$. The same tree describes the respective complex zero-dimensional stratum of $\overline{\mathcal{M}}_{0, S}$.

Each boundary divisor containing such a stratum can be obtained by choosing an interior edge of $\tau$ : if we cut the edge, $S$ will break into two parts corresponding to the distribution of tails among the resulting connected components of $\tau$. Clearly, the same 2-partition of $S$ can be obtained by breaking the circle into two connected arcs.

It remains to check that such a partition cannot have a definite type with respect to $\underline{\varepsilon}$. In other words, no arc can contain only points of $S$ forming a set $\{\alpha\} \cup T, T \subset S(\alpha)$. In fact, for $\alpha=\infty$ this is impossible, because 1 and 0 separate $\infty$ from any element $s_{i}$. For $\alpha=0$ this is impossible because $s_{1} \in S(1)$ and $\infty$ separate 0 from any element of $S(0)$. The case $\alpha=1$ is impossible for a similar reason.

\subsection{Proof of Proposition 3.1}

We will argue by induction on $n$.

Case $n=1$. Here $S=\{0,1, \infty, s\}$. As we recalled in $\S 2, \overline{\mathcal{M}}_{0, S}$ is a projective line with three boundary divisors corresponding to the partitions $s 0|1 \infty, s 1| 0 \infty$, and $s \infty \mid 01$. Moreover, $t:=t_{s}$ is the affine coordinate on this projective line taking, respectively, the values 0,1 , and $\infty$ at these three boundary divisors. A form $d t /(t-\varepsilon), \varepsilon=0,1$, is singular at two partitions: $s \varepsilon \mid(1-\varepsilon) \infty$ and $s \infty \mid 01$. Comparing this with the definition in Subsection 3.1, we see that these are exactly those partitions that have a definite type with respect to $\varepsilon$.

Case $n=2$. Although it is not strictly necessary to treat this case separately, it will be convenient to do so: we will show both the essence of the inductive step and the geometry of the first simple but non-trivial situation.

We put $S=\left\{0,1, \infty, s_{1}, s_{2}\right\}, t_{i}=t_{s_{i}}$, and choose $\varepsilon_{1}=1, \varepsilon_{0}=0$. For $n=2$, this is the only case producing a $\zeta$-value, namely $\zeta(2)$. 


\section{Multiple $\zeta$-Motives And moduli spaces $\overline{\mathcal{M}}_{0, n}$}

Two morphisms forgetting $x_{s_{2}}$ and $x_{s_{1}}$ respectively, represent $\overline{\mathcal{M}}_{0, S}$ as a blow-up of a quadric:

$$
\beta: \overline{\mathcal{M}}_{0, S} \rightarrow \overline{\mathcal{M}}_{0,\left\{0,1, \infty, s_{1}\right\}} \times \overline{\mathcal{M}}_{0,\left\{0,1, \infty, s_{2}\right\}} \cong \mathbf{P}^{1} \times \mathbf{P}^{1}
$$

In coordinates $\left(t_{1}, t_{2}\right)$ three points are blown: $(0,0),(1.1)$, and $(\infty, \infty)$. The resulting divisors are (correspond to), respectively, the following partitions of $S: s_{1} s_{2} 0\left|1 \infty, s_{1} s_{2} 1\right| 0 \infty$, and $s_{1} s_{2} \infty \mid 01$. The strict preimage of the diagonal is the boundary divisor $s_{1} s_{2} \mid 01 \infty$. All in all, there are ten boundary components. We split them into a union of two 'pentagons'. The pentagon $A=A(1,0)$ consists of the strict preimages of the divisors $t_{1}=1, t_{1}=\infty, t_{2}=0, t_{2}=\infty$, and of the blow-up of $(\infty, \infty)$.

This is the picture we referred to in $\S 1$.

It is important to understand why the pentagon $A$ is precisely the divisor of singularities of

$$
\Omega(1,0)=\beta^{*}\left(\frac{d t_{1}}{t_{1}-1} \wedge \frac{d t_{2}}{t_{2}}\right) .
$$

The point is that if we naively add up the total preimages of singularities of $d t_{1} /\left(t_{1}-1\right)$ and $d t_{2} / t_{2}$, we will get two spurious extra components: blow-ups of $(0,0)$ and $(1,1)$. A local calculation shows that they cancel in $\Omega(1,0)$, because neither $(0,0)$ nor $(1,1)$ is a stratum of the divisor of singularities of the form on the quadric.

It is this step that is crucial - and somewhat cumbersome to check - in the general inductive reasoning.

To complete the discussion of our example, notice that the complementary pentagon, which is the union of the strict preimages of $t_{1}=0, t_{1}=t_{2}, t_{2}=1$ and the blow-ups of $(0,0)$ and $(1,1)$, is precisely $B$. The reader may check it as a nice exercise.

General inductive step. Let $S=\left\{s_{1}, \ldots, s_{n}\right\}, S^{\prime}=\left\{s_{1}, \ldots, s_{n}, s_{n+1}\right\}, \varepsilon_{s_{i}}=\varepsilon_{i}, \underline{\varepsilon}=\left\{\varepsilon_{1}, \ldots, \varepsilon_{n}\right\}$, and $\underline{\varepsilon}^{\prime}=\left\{\varepsilon_{1}, \ldots, \varepsilon_{n+1}\right\}$. Generalizing (8), we define a birational morphism composed of two forgetful maps

From (7), it is clear that

$$
\beta: \overline{\mathcal{M}}_{0, S^{\prime}} \rightarrow \overline{\mathcal{M}}_{0, S} \times \overline{\mathcal{M}}_{0,\left\{0,1, \infty, s_{n+1}\right\}}
$$

$$
\Omega\left(\underline{\varepsilon}^{\prime}\right)=\beta^{*}\left(\Omega(\underline{\varepsilon}) \wedge \frac{d t_{n+1}}{t_{n+1}-\varepsilon_{n+1}}\right) .
$$

The divisor of (logarithmic) singularities of $\Omega(\underline{\varepsilon}) \wedge d t_{n+1} /\left(t_{n+1}-\varepsilon_{n+1}\right)$ is a normal crossing divisor consisting of (lifts of) some boundary divisors of the factors. More precisely, by the inductive assumption, the latter constitute $A\left(\varepsilon_{n+1}\right)$ and $A(\underline{\varepsilon})$, respectively.

Hence the divisor of $\Omega\left(\underline{\varepsilon}^{\prime}\right)$ is $\beta^{*}\left(\operatorname{pr}_{1}^{*} A(\underline{\varepsilon})+\operatorname{pr}_{2}^{*} A\left(\varepsilon_{n+1}\right)\right)$ minus eventual spurious components.

According to [Gon02, Lemma 3.8], the spurious components are those irreducible boundary divisors in $\overline{\mathcal{M}}_{0, S^{\prime}}$ which get blown down by $\beta$ to a subvariety of the product which is not a stratum of the divisor $\operatorname{pr}_{1}^{*} A(\underline{\varepsilon})+\operatorname{pr}_{2}^{*} A\left(\varepsilon_{n+1}\right)$. Let us now make explicit the combinatorics of the situation. We will systematically identify boundary divisors with partitions.

i) First of all, a boundary divisor of $\overline{\mathcal{M}}_{0, S^{\prime}}$ gets blown down by $\beta$ if both forgetful morphisms map it into boundary divisors. Combinatorially, we take a stable partition of $S^{\prime}$ and induce from it a partition of $S$ and of $\left\{0,1, \infty, s_{n+1}\right\}$; both results must be stable. Contemplation will convince the reader that there are three kinds of such partitions:

$$
s_{n+1} 0 \sigma_{1}\left|\sigma_{2} 1 \infty, \quad s_{n+1} 1 \sigma_{1}\right| \sigma_{2} 0 \infty, \quad s_{n+1} \infty \sigma_{1} \mid \sigma_{2} 01,
$$

where $\sigma_{1} \mid \sigma_{2}$ is any partition of $\left\{s_{1}, \ldots, s_{n}\right\}$ with $\sigma_{1} \neq \emptyset$.

Moreover, divisors $s_{n+1} \sigma_{1} \mid \sigma_{2} 01 \infty$ with $\left|\sigma_{1}\right| \geqslant 2$ also get blown down, and this completes the list: see [Kee92, Lemma 1, p. 554]. 


\section{A. B. Goncharov and Yu. I. Manin}

ii) Now let us list the components of $A(\underline{\varepsilon})$. They are also divided into three types (see the beginning of this section):

$$
0 T_{0}\left|\ldots 1 \infty, \quad 1 T_{1}\right| \ldots 0 \infty, \quad \infty T_{\infty} \mid \ldots 01
$$

where $T_{\alpha}$ is a non-empty subset of $S(\alpha)$. Each of them has exactly two strict lifts to $\overline{\mathcal{M}}_{0, S^{\prime}}$ : we may add $s_{n+1}$ to the first or to the second parts of the partition. We will first check which of these lifts do not belong to $A\left(\underline{\varepsilon}^{\prime}\right)$. Assume for concreteness that $\varepsilon_{n+1}=0$; the other case is treated similarly.

Again, contemplation will convince the reader that only the following lifts do not have a definite type with respect to $\underline{\varepsilon}^{\prime}$ :

$$
s_{n+1} 1 T_{1} \mid \ldots 0 \infty \text {. }
$$

iii) Let us now check that the components (13) are spurious. First, forgetting $s_{n+1}$ or all $\left\{s_{1}, \ldots, s_{n}\right\}$ produces stable partitions, so such a divisor gets blown down. More precisely, its $\beta$-image is (and does not just lie in)

$$
1 T_{1}\left|\ldots 0 \infty \times s_{n+1} 1\right| 0 \infty .
$$

This product is not a stratum of the divisor of $\Omega(\underline{\varepsilon}) \wedge d t_{n+1} / t_{n+1}$. In fact, the first factor is a component of the singularities of $\Omega(\underline{\varepsilon})$, whereas the second one is not a singularity for $d t_{n+1} / t_{n+1}$.

iv) After adding $s_{n+1}$ to one of the parts of partitions in the list (12) and deleting the partitions (13), we will get almost all partitions of $S^{\prime}$ having a definite type with respect to $\underline{\varepsilon}^{\prime}$. The only exceptions will be (recall that $\varepsilon_{n+1}=0$ )

$$
s_{n+1} 0\left|S 1 \infty, \quad s_{n+1} \infty\right| S 01 .
$$

These two components of $A\left(\underline{\varepsilon}^{\prime}\right)$ are supplied by the lifts of the poles of $d t_{n+1} / t_{n+1}$.

This concludes the proof.

\section{Multiple $\zeta$-motives}

\subsection{Motivic background}

In the following we employ the abelian category $\mathcal{M}_{T}(\mathbb{Q})$ of mixed Tate motives over $\mathbb{Q}$ as defined in [Gon99, ch. 5], (see also [Lev93]), and its subcategory $\mathcal{M}_{T}(\mathbb{Z})$ of mixed Tate motives unramified over $\mathbb{Z}[\mathrm{DG}]$. As is shown in loc. cit., an object of $\mathcal{M}_{T}(\mathbb{Q})$ is unramified over $\mathbb{Z}$ if and only if for any prime $l$ its $l$-adic realization is unramified outside of $l$.

To talk about the periods of mixed Tate motives we need to equip mixed Tate motives with an additional structure called framing. For a definition of framed mixed Tate objects, e.g. framed mixed Tate motives or framed Hodge-Tate structures, see for example [Gon01b, § 3.2]. To define a period of a framed Hodge-Tate structure $H$ we need to choose a splitting of the weight filtration on $H_{\mathbb{Q}}$, see the next section and [Gon99, Subsection 4.2] for more details.

\subsection{Multiple $\zeta$-motives}

Keeping the notation of Subsection 2.1, we define

$$
\zeta^{\mathcal{M}}\left(n_{1}, \ldots, n_{m}\right):=H^{n}\left(\overline{\mathcal{M}}_{0, n+3}-A(\varepsilon), B-B \cap A(\varepsilon)\right) .
$$

\section{Theorem 4.1.}

i) $\zeta^{\mathcal{M}}\left(n_{1}, \ldots, n_{m}\right)$ is a mixed Tate motive unramified over $\operatorname{Spec}(\mathbb{Z})$.

ii) $\Omega(\underline{\varepsilon})$ and $\Phi(\rho)$ allow us to define its natural framing so that the respective period equals $\zeta\left(n_{1}, \ldots, n_{m}\right)$. 


\section{Multiple $\zeta$-Motives And moduli spaces $\overline{\mathcal{M}}_{0, n}$}

Proof. A non-singular projective variety is called a Tate variety if its motive is a direct sum of pure Tate motives. It is well-known (and follows from Knudsen's inductive description of $\overline{\mathcal{M}}_{0, n+3}$ ) that all strata of the stratification defined by the divisor $\partial \overline{\mathcal{M}}_{0, n+3}$ are Tate varieties. Since $\partial \overline{\mathcal{M}}_{0, n+3}$ is a normal crossing divisor, Corollary 3.2 above and [Gon02, Proposition 3.6] show that $\zeta^{\mathcal{M}}\left(n_{1}, \ldots, n_{m}\right)$ is well-defined as a mixed Tate motive over $\mathbb{Q}$.

Let us recall briefly its construction. Consider the standard cosimplicial variety

$$
S_{\bullet}\left(\overline{\mathcal{M}}_{0, n+3}-A(\underline{\varepsilon}), B\right)
$$

corresponding to the pair $\left(\overline{\mathcal{M}}_{0, n+3}-A(\underline{\varepsilon}), B-B \cap A(\underline{\varepsilon})\right)$.

Recall that $S_{0}:=\overline{\mathcal{M}}_{0, n+3}-A(\underline{\varepsilon})$, and $S_{k}$ is the disjoint union of the codimension $k$ strata of the divisor $B-B \cap A(\underline{\varepsilon})$.

Following the standard procedure, let us make a complex of varieties out of (15) with $S_{0}$ placed at the degree zero. It provides an object in the Voevodsky triangulated category of motives [Voe00], which in fact belongs to the triangulated subcategory $\mathcal{D}_{T}(\mathbb{Q})$ of mixed Tate motives over $\mathbb{Q}$. There exists a canonical $t$-structure $t$ on the category $\mathcal{D}_{T}(\mathbb{Q})$ (see [Gon99, ch. 5] or [Lev93] for its definition). Then $H_{t}^{n}$ of the above complex is the multiple $\zeta$-motive.

Now we will introduce a framing. Generally, a framing on a mixed motive is an algebraic counterpart of the notion of period. For the mixed Tate motives it is as follows. Recall that a mixed Tate motive carries a canonical weight filtration $W_{\bullet}$ such that the associated graded motive of weight $-2 k$ is a direct sum of pure Tate motives $\mathbb{Q}(k)$, and $\operatorname{Gr}_{2 k-1}^{W}=0$. An $n$-framed (or simply framed if $n$ is clear from the context) mixed Tate motive is a triple $(M, v, f)$, where $v$ and $f$ are two non-zero morphisms

$$
v: \mathbb{Q}(-n) \rightarrow \mathrm{Gr}_{2 n}^{W} M, \quad f: \mathbb{Q}(0) \rightarrow\left(\mathrm{Gr}_{0}^{W} M\right)^{\vee}=\mathrm{Gr}_{0}^{W} M^{\vee},
$$

and $M^{\vee}$ is the dual object.

In our situation we define a framing as follows. There are canonical isomorphisms

$$
\operatorname{Gr}_{2 n}^{W} H^{n}\left(\overline{\mathcal{M}}_{0, n+3}-A(\underline{\varepsilon}), B-B \cap A(\varepsilon)\right) \cong \operatorname{Gr}_{2 n}^{W} H^{n}\left(\overline{\mathcal{M}}_{0, n+3}-A(\underline{\varepsilon})\right)
$$

and

$$
\mathrm{Gr}_{0}^{W} H_{n}\left(\overline{\mathcal{M}}_{0, n+3}-A(\underline{\varepsilon}), B-B \cap A(\varepsilon)\right) \cong \mathrm{Gr}_{0}^{W} H_{n}\left(\overline{\mathcal{M}}_{0, n+3}, B\right) .
$$

Moreover, there are natural non-zero morphisms of pure Tate motives

$$
\begin{gathered}
{[\Omega(\underline{\varepsilon})]: \mathbb{Q}(-n) \rightarrow \operatorname{Gr}_{2 n}^{W} H^{n}\left(\overline{\mathcal{M}}_{0, n+3}-A(\underline{\varepsilon})\right),} \\
{\left[\Phi_{n}\right]: \mathbb{Q}(0) \rightarrow \operatorname{Gr}_{0}^{W} H_{n}\left(\overline{\mathcal{M}}_{0, n+3}, B\right) .}
\end{gathered}
$$

To construct them we employ the fact that the Hodge realization is a fully faithful functor on the category of pure Tate motives. Then the first morphism is determined in the De Rham realization by the form $\Omega(\underline{\varepsilon})$. The second morphism is determined in the Betti realization by the relative homology class of the cell $\bar{\Phi}_{n}$. Combining them with the isomorphisms (16) and (17) we get the frame morphisms

$$
\begin{gathered}
{[\Omega(\underline{\varepsilon})]^{\prime}: \mathbb{Q}(-n) \rightarrow \mathrm{Gr}_{2 n}^{W} H^{n}\left(\overline{\mathcal{M}}_{0, n+3}-A(\underline{\varepsilon}), B-B \cap A(\varepsilon)\right),} \\
{\left[\Phi_{n}\right]^{\prime}: \mathbb{Q}(0) \rightarrow \operatorname{Gr}_{0}^{W} H_{n}\left(\overline{\mathcal{M}}_{0, n+3}-A(\underline{\varepsilon}), B-B \cap A(\varepsilon)\right) .}
\end{gathered}
$$

Let us show that $\zeta\left(n_{1}, \ldots, n_{m}\right)$ is a period of this framed mixed Tate motive.

Recall that a mixed Hodge structure is the following linear algebra data:

i) a $\mathbb{Q}$-vector space $H_{\mathbb{Q}}$ equipped with an increasing filtration $W_{\bullet}$;

ii) a decreasing filtration $F^{\bullet}$ on $H_{\mathbb{C}}=H_{\mathbb{Q}} \otimes \mathbb{C}$ inducing, for each integer $n$, a weight $n$ pure Hodge 


\section{A. B. Goncharov and Yu. I. Manin}

structure on $\mathrm{Gr}_{n}^{W} H$, i.e.

$$
\operatorname{Gr}_{n}^{W} H_{\mathbb{C}}=\oplus_{p+q=n} F^{p} \operatorname{Gr}_{n}^{W} H_{\mathbb{C}} \cap \bar{F}^{q} \operatorname{Gr}_{n}^{W} H_{\mathbb{C}}
$$

A Hodge-Tate structure is a mixed Hodge structure with the Hodge numbers $h^{p q}=0$ unless $p=q$. It is easy to see that this is equivalent to the following condition: for every $p \in \mathbb{Z}$ the natural map

is an isomorphism.

$$
F^{p} H_{\mathbb{C}} \cap W_{2 p} H_{\mathbb{C}} \rightarrow \mathrm{Gr}_{2 p}^{W} H_{\mathbb{C}}
$$

The Hodge realization of the multiple $\zeta$-motive is a Hodge-Tate structure.

An $n$-framed Hodge-Tate structure is a Hodge-Tate structure $H$ equipped with non-zero morphisms

$$
v: \mathbb{Q}(-n) \rightarrow \mathrm{Gr}_{2 n}^{W} H, \quad f: \mathbb{Q}(0) \rightarrow\left(\mathrm{Gr}_{0}^{W} H\right)^{\vee}=\mathrm{Gr}_{0}^{W} H^{\vee} .
$$

To define a period of a framed Hodge-Tate structure we need to choose additional data - a map of $\mathbb{Q}$-vector spaces $\widetilde{f}: \mathbb{Q} \rightarrow H_{\mathbb{Q}}^{\vee}$ lifting $f$, i.e. $\operatorname{Gr}_{0}^{W} \widetilde{f}=f$. Set $f^{\prime}:=\widetilde{f}(1)$. The composition

$$
\mathbb{Q}(-n) \rightarrow \operatorname{Gr}_{2 p}^{W} H_{\mathbb{Q}} \rightarrow F^{p} H_{\mathbb{C}} \cap W_{2 p} H_{\mathbb{C}}
$$

where the first arrow is $v$ and the second arrow is provided by (18), leads to a vector $v^{\prime} \in F^{p} H_{\mathbb{C}} \cap$ $W_{2 p} H_{\mathbb{C}}$. The corresponding period is a complex number $\left\langle v^{\prime}, f^{\prime}\right\rangle$. A different choice of lifting $\widetilde{f}$ changes the period by $2 \pi i$ times a 'weight $n-1$ period', see [Gon99, Subsection 4.2] for more details.

In our case a canonical choice of the lift $\widetilde{f}$ is secured by Corollary 3.2 which shows that the cell $\bar{\Phi}_{n}$ provides an element of the Betti homology

$$
\left[\bar{\Phi}_{n}\right]^{\prime} \in H_{n}^{B}\left(\overline{\mathcal{M}}_{0, n+3}-A(\underline{\varepsilon}), B-B \cap A(\underline{\varepsilon})\right) .
$$

The restriction of the form $\Omega(\underline{\varepsilon})$ to the divisor $B$ is zero, so that it furnishes a De Rham cohomology class

$$
[\Omega(\underline{\varepsilon})]^{\prime} \in H_{\mathrm{DR}}^{n}\left(\overline{\mathcal{M}}_{0, n+3}-A(\underline{\varepsilon}), B-B \cap A(\underline{\varepsilon})\right) .
$$

These are the classes obtained before by using isomorphisms (16) and (17). Clearly, the integral (5) computes the pairing of these two classes.

It remains to show that $\zeta^{\mathcal{M}}\left(n_{1}, \ldots, n_{m}\right)$ is unramified over $\mathbb{Z}$.

Definition 4.2. Let $D$ be a normal crossing divisor in a regular scheme $X$ over $\mathbb{Z}_{p}$. Assume that the pair $(D, X)$ is proper over $\mathbb{Z}_{p}$. We say that reduction modulo $p$ does not change the combinatorics of $(D, X)$ if $X$ and every stratum of $D$ are smooth over $\mathbb{Z}_{p}$, and the reduction map from the strata of $D$ to ones at the special fiber is a bijection.

Let $X$ be a flat integral scheme of finite type over $\mathbb{Z}_{p}$, with $D$ a divisor. Set $\bar{X}:=X \otimes_{\mathbb{Z}_{p}} \overline{\mathbb{Q}}_{p}$. Let $A$ and $B$ be the unions of two disjoint subsets of irreducible components of $D$.

Proposition 4.3. If the reduction modulo $p$ does not change the combinatorics of $(D, X)$ and $(l, p)=1$, then the $\operatorname{Gal}\left(\overline{\mathbb{Q}}_{p} / \mathbb{Q}_{p}\right)$-module $H_{\text {et }}^{n}\left(\bar{X}-\bar{A}, \bar{B}-\bar{B} \cap \bar{A} ; \mathbb{Q}_{l}\right)$ is unramified at $p$.

Proof. It is proved by taking the standard simplicial resolution computing $H^{n}(X-A, B-B \cap A)$ and using the proper and smooth base change theorem, see [Gon02, Proposition 3.9] for details.

Proposition 4.4. For any prime $p$ the reduction modulo $p$ does not change the combinatorics of the divisor $\partial \overline{\mathcal{M}}_{0, n+3}$.

In fact, the description of the combinatorics of the boundary strata given in Subsection 2.2 is valid over a field of any characteristic $p$ and is compatible with reduction.

This concludes the proof of Theorem 4.1. 


\subsection{Concluding remarks}

The above discussion can be applied to more general pairs $A, B$ sharing no common components for which we can find framing as in (3). For example, interchanging $A$ and $B$ and using the duality isomorphism

$$
H^{n}\left(\overline{\mathcal{M}}_{0, n+3}-A\right)^{\vee} \cong H_{n}\left(\overline{\mathcal{M}}_{0, n+3}, A\right)(n),
$$

which implies

$$
\operatorname{Gr}_{2 n}^{W} H^{n}\left(\overline{\mathcal{M}}_{0, n+3}-A\right) \cong \operatorname{Gr}_{0}^{W} H_{n}\left(\overline{\mathcal{M}}_{0, n+3}, A\right)(n),
$$

we get a different mixed Tate motive over $\mathbb{Z}$ with the dual framing. Hence we get a concrete problem: Problem. Let $\widehat{\zeta}^{\mathcal{M}}\left(n_{1}, \ldots, n_{m}\right)$ be the mixed Tate motive obtained by interchanging the $A$ - and $B$-divisors in (14). Express $\widehat{\zeta}^{\mathcal{M}}\left(n_{1}, \ldots, n_{m}\right)$ via the multiple $\zeta$-motives.

According to [Gon95, Conjecture 17(b)] any framed mixed Tate motive unramified over $\mathbb{Z}$ is equivalent to a linear combination of framed multiple $\zeta$-motives. In our situation, we come to the following conjecture.

Conjecture 4.5. Any mixed Tate motive (2) with a framing provided by (3) is equivalent to a $\mathbb{Q}$-linear combination of the framed weight $n$ multiple $\zeta$-motives.

The analytic version of this conjecture says that, for any $\left[\Delta_{B}^{\prime}\right] \in H_{n}\left(\overline{\mathcal{M}}_{0, n+3}-A, B-B \cap A\right)$ lifting $\left[\Delta_{B}\right] \in \operatorname{Gr}_{0}^{W} H_{n}\left(\overline{\mathcal{M}}_{0, n+3}, B\right)$ in (3), the integral $(2 \pi i)^{-n} \int_{\Delta_{B}^{\prime}} \omega_{A}$ giving a period of the framed Hodge-Tate structure $(4)$ is $(2 \pi i)^{-n} \times$ (a $\mathbb{Q}$-linear combination of the weight $n$ multiple $\zeta$-values).

\subsection{Generalizations}

Consider an iterated integral

$$
\mathrm{I}\left(a_{1}, \ldots, a_{n}\right):=\int_{\Delta_{n}^{0}} \frac{d t_{1}}{t_{1}-a_{1}} \wedge \cdots \wedge \frac{d t_{n}}{t_{n}-a_{n}}, \quad a_{1} \neq 0, a_{n} \neq 1 .
$$

Let $\underline{a}:=\left\{a_{1}, \ldots, a_{n}\right\}$ and $A(\underline{a})$ be the divisor of singularities of the form

$$
\Omega(\underline{a}):=\frac{d t_{1}}{t_{1}-a_{1}} \wedge \cdots \wedge \frac{d t_{n}}{t_{n}-a_{n}}
$$

meromorphically extended to $\overline{\mathcal{M}}_{0, n+3}$. Then extending the definitions above in an obvious way, one can prove the following.

Proposition 4.6. The divisor $A(\underline{a})$ contains no faces of the divisor $B$.

The absence of common components follows immediately from the convergence of the integral (19).

Thus if $a_{i}$ are elements of a number field $F$ then

$$
H^{n}\left(\overline{\mathcal{M}}_{0, n+3}-A(\underline{a}), B-B \cap A(\underline{a})\right)
$$

is a mixed Tate motive over $F$. The class $\left[\Delta_{B}\right]$ and the form $\Omega(\underline{a})$ provide it with a framing. If $a_{i}$ are complex numbers then the period of the corresponding framed Hodge-Tate structure is given by the integral $\mathrm{I}\left(a_{1}, \ldots, a_{n}\right)$.

This provides a different construction of the motivic multiple polylogarithms motives then the one suggested in [Gon02]. However, the framed mixed Tate motives given by these two constructions are equivalent. 


\section{Multiple $\zeta$-Motives ANd moduli spaces $\overline{\mathcal{M}}_{0, n}$}

\section{ACKNOWLEDGEMENTS}

This paper was conceived at the MPIM (Bonn) in June of 1997, and its rough draft was prepared during the Fall of 2001 when A. G. visited MSRI (Berkeley). A. G. is very grateful to both institutions for providing ideal working conditions. We are grateful to V. Fock for useful discussions.

\section{REFERENCES}

Del P. Deligne, Resumé des premiers exposés de A. Grothendieck, in Groupes de monodromie en géométrie algébrique, SGA 7, 1, Lecture Notes in Mathematics, vol. 288, 1-24 (Springer-Verlag, Berlin, 1972).

DG P. Deligne and A. B. Goncharov, Groupes fondamentaux motiviques de Tate mixte, math.NT/0302267.

Dev99 S. L. Devadoss, Tesselations of moduli spaces and the mosaic operad, Contemp. Math. 239 (1999), 91-114.

Dri91 V. G. Drinfeld, On quasitriangular quasi-Hopf algebras and on a group that is closely connected with $\mathrm{Gal}(\overline{\mathbb{Q}} / \mathbb{Q})$, Leningrad Math. J. 2(4) (1991), 829-860.

Eul17 L. Euler, Opera omnia, Ser. 1, Vol. XV (Teubner, Berlin 1917), 217-267.

Gon95 A. B. Goncharov, Polylogarithms in arithmetic and geometry, in Proc. int. congress of mathematicians, Zürich, 1994, vol. 1, 2 (Birkhäuser, Basel, 1995), 374-387.

Gon99 A. B. Goncharov, Volumes of hyperbolic manifolds and mixed Tate motives, J. Amer. Math. Soc. (2) 12 (1999), 569-618, math.alg-geom/9601021.

Gon01a A. B. Goncharov, Multiple $\zeta$-values, Galois groups and geometry of modular varieties in Proc. third European congress of mathematicians, Progress in Mathematics, vol. 201 (Birkhäuser, Basel, 2001), 361-392, math.AG/0005069.

Gon01b A. B. Goncharov, Multiple polylogarithms and mixed Tate motives, math.AG/0103059.

Gon02 A. B. Goncharov, Periods and mixed motives, math.AG/0202154.

Kap93 M. M. Kapranov, The permutoassociahedron, MacLane's coherence theorem and asymptotic zones for the KZ equation. J. Pure Appl. Algebra 85 (1993), 119-142.

Kee92 S. Keel, Intersection theory of moduli space of stable $N$-pointed curves of genus zero, Trans. Amer. Math. Soc. 330(2) (1992), 545-574

Knu83 F. F. Knudsen, The projectivity of the moduli space of stable curves II. The stacks $\bar{M}_{0, n}$, Math. Scand. 52 (1983), 163-199.

Man99 Yu. I. Manin, Frobenius manifolds, quantum cohomology, and moduli spaces, AMS Colloquium Publications, vol. 47 (American Mathematical Society, Providence, RI, 1999), 303.

Lev93 M. Levine, Tate motives and the vanishing conjectures for algebraic K-theory, in Algebraic K-theory and algebraic topology, Lake Louise, AB, 1991, NATO Adv. Sci. Inst. Ser. C Math. Phys. Sci., vol. 407 (Kluwer Academic, Dordrecht, 1993), 167-188.

Ter01 T. Terasoma, Multiple zeta values and mixed Tate motives, math.AG/0104231.

Voe00 V. Voevodsky, Triangulated category of motives over a field, in Cycles, transfers, and motivic homology theories, Annals of Math. Studies, vol. 143 (Princeton University Press, Princeton, NJ, 2000), 188-238.

A. B. Goncharov sasha@math.brown.edu

Department of Mathematics, Brown University, Providence, RI 02912, USA

Yu. I. Manin manin@mpim-bonn.mpg.de

Max-Planck-Institut für Mathematik, Vivatsgasse 7, Bonn, Germany 